A N N A L E S Annales de Bretagne et des Pays de l'Ouest

\title{
Les Bretons et la Grande Guerre
}

\section{Ronan Richard}

\section{OpenEdition}

\section{Journals}

Édition électronique

URL : http://journals.openedition.org/abpo/2816

DOI : $10.4000 / a b p o .2816$

ISBN : 978-2-7535-3513-8

ISSN : 2108-6443

\section{Éditeur}

Presses universitaires de Rennes

Édition imprimée

Date de publication : 30 juin 2014

ISBN : 978-2-7535-3511-4

ISSN : 0399-0826

Référence électronique

Ronan Richard, "Les Bretons et la Grande Guerre », Annales de Bretagne et des Pays de l'Ouest [En ligne], 121-2 | 2014, mis en ligne le 30 juin 2014, consulté le 23 septembre 2020. URL : http:// journals.openedition.org/abpo/2816; DOI : https://doi.org/10.4000/abpo.2816 
des putains. " - " Baube, chef de bureau à la Préfecture de Police, conte à Alphonse que $\mathrm{M}^{\mathrm{me}}$ la princesse de Chimay est tarifée $5000 \mathrm{~F}$ pour une nuit. " Cette crudité se combine à une sensualité très marquée qu'on retrouve à de nombreuses reprises. En 1891 (p. 130) : "Le concours de nuque n'a pas été vraiment épatant, il y a eu onze femmes et la nuque s'étendait jusqu'au commencement des fesses. Quelquesunes étaient bien. La nuit s'est terminée sans orgie, calme. Du pelotage, mais pas de femmes mises nues. On n'osait pas et on l'aurait bien voulu ". Ce goût du détail ne se limite d'ailleurs pas aux questions de mœurs ou de corruption. Il arrive souvent à Hamon de donner des précisions infimes sur le prix des objets ou des services, ce qui n'est pas sans intérêt. Sa difficile situation financière, difficile et permanente, l'avait sans doute amené à manifester beaucoup d'attention à ces questions.

Remarquons que ce souci du détail n'empêche pas Hamon de donner ici ou là ses impressions sur la situation politique générale. On voit ainsi défiler des considérations sur la politique britannique en Irlande. Les prévisions concernant la situation en Russie (p. 416, 439, et autres) sont dans l'ensemble très perspicaces. Le récit du second procès Dreyfus à Rennes (p. 362-365) est du plus haut intérêt : Hamon n'y était pas mais il lui fut fait par l'éditeur Stock avec qui il était en relations. On ne peut savoir si, comme il le prétend, Waldeck-Rousseau était persuadé de l'acquittement ou s'il jouait un jeu tortueux peu surprenant de la part du personnage. Fort captivants aussi, les relents antisémites nombreux sous la plume d'Hamon, très répandus dans l'extrême gauche française jusqu'à l'" Affaire " et, contrairement à ce que l'on dit parfois, au-delà. Les propos venimeux rapportés contre Joseph Reinach et la famille Dreyfus ne sont qu'un des multiples témoignages de cet état d'esprit.

Le texte est présenté de façon claire et annoté par Patrick Galliou. Les notes tentent de situer le maximum de personnages évoqués, mais, comme on l'a dit, un nombre important, par force, restent dans l'ombre. Certaines notes - sans doute était-ce également peu évitable - sont un peu surprenantes ou inexactes. Sur Quesnay de Beaurepaire (p. 90) : «En 1893, il était Président de la Chambre de cassation [sic] et il fut l'accusateur de Dreyfus". Quesnay était président d'une des chambres civiles de la Cour de cassation et il ne fut pas "l'accusateur de Dreyfus". Ce n'est que bien plus tard, et par pur dépit personnel, qu'il rejoignit le camp antidreyfusard dont il fut toujours un membre assez peu pris au sérieux aussi bien par ses " amis " que par ses ennemis. La note sur J.H. Rosny aîné, ce grand écrivain un peu oublié (p. 117) est très réductrice et classer Le Bilatéral, roman naturaliste sur le peuple de Paris, parmi les romans " préhistoriques " témoigne d'une rédaction hâtive. La note sur Charles Richet (p. 523) ne rend pas vraiment justice à ce personnage étonnant et complexe. Etc.

Au total, un texte du plus grand intérêt et d'un ton très inhabituel dont la publication se révèle d'une haute utilité.

Jean-François TANGUY

GuYvarc'H, Didier, Lagadec, Yann, Les Bretons et la Grande Guerre, Rennes, PUR, 2013, $208 \mathrm{p}$.

Traditionnellement, les années commémoratives constituent des effets d'aubaine qui suscitent toujours une incroyable inflation de publications d'une qualité scientifique pour le moins inégale. Le centenaire du déclenchement de la Première guerre mondiale ne fait pas exception à la règle si l'on en juge par le foisonnement d'ouvrages en tout genre qui, sans attendre l'été 2014, inondent déjà par anticipation les étals des libraires. Pour autant, ce centenaire constitue aussi un rendez-vous 
pédagogique capital entre historiens et grand public, l'engouement médiatique rendant indispensable une mise au point rigoureuse et documentée, ne serait-ce que pour contrecarrer les ouvrages où l'intérêt lucratif le dispute au sérieux du contenu. C'est le défi ambitieux auquel s'attèle cet ouvrage. Pour premier atout, et non des moindres, cette mission a été confiée à des plumes qualifiées dont l'expérience sur le sujet est incontestable. Ajoutons que cet ouvrage est préfacé par Alain Croix dont la grande rigueur achève de nous parer contre tout soupçon de publication de pure circonstance.

D'emblée, l'ouvrage affiche son angle d'attaque méthodologique. Publié dans la collection "Images et histoires ", il privilégie le recours à cette image qui informe ou qui déforme, qui témoigne ou qui manipule, une image porteuse de sens car toujours replacée dans son contexte général. Le panel iconographique offre une lecture de l'histoire riche, variée et équilibrée (cartes postales, journaux, peintures, dessins et croquis, photographies privées ou institutionnelles, affiches, archives, monuments, vitraux...), étayée par de nombreux témoignages émanant du terrain. L'intérêt de cette démarche réside d'abord dans la forme. L'ouvrage est au premier abord un bel objet, agréable à compulser et dont les illustrations permettent une entrée facile dans la lecture. Ce choix n'est certes pas sans écueil. En effet, partir de l'image aboutit parfois à réduire l'histoire à ses aspects purement matériels et conduit à faire l'impasse sur ces autres pans plus immatériels et invisibles que constituent les représentations, les sentiments ou les mutations culturelles. Le grand mérite des auteurs est d'avoir justement su contourner finement cet obstacle et faire disparaître au gré des pages cette crainte et ces réserves d'ordre méthodologique, dressant par exemple un tableau très complet de la vie matérielle du combattant mais aussi de la culture du front, du rapport à la mort, des solidarités, des expédients pour tenir et lutter contre le cafard, des peurs, des doutes et des défaillances aussi (fraternisations, folie, suicide, mutinerie). La problématique générale pose la question d'une hypothétique "façon d'être Breton dans la guerre ". De fait, les auteurs ne découvrent guère de particularisme régional dans les comportements ni les représentations durant la guerre. Les mobilisés partent comme partout en France, sans enthousiasme, graves mais résolus. L'engagement et la ténacité légendaires du soldat breton relèvent moins du constat réel que d'une image héroïsante sciemment conçue pour "faire tenir ". Certes, l'appartenance à la petite Patrie et la pratique linguistique nouent des solidarités et le combattant breton reste ancré à ses symboles culturels et à sa foi catholique qui constituent de réels marqueurs régionaux. Pour autant, l'identité régionale bretonne se trouve globalement noyée dans le brassage régimentaire dès la fin de l'année 1914. À l'arrière, la spécificité de la Bretagne tient à sa situation géographique. Terre d'ouverture par ses ports, terre de repli militaire et sanitaire, terre d'accueil des réfugiés et prisonniers, elle connaît par ailleurs les mêmes mutations que le reste de la France : l'adaptation fébrile à l'économie de guerre, la crise de la main-d'œuvre, les conflits sociaux... Quant au recours aux " bretonnités", stéréotypes culturels d'une "Bretagne pour veillée de chaumière ", il témoigne moins d'une identité régionale revigorée que d'une instrumentalisation par les autorités au service de l'intégration et de l'unité nationale. Le titre même de l'ouvrage révèle enfin un autre angle d'attaque très pertinent : les auteurs ne traitent pas des Bretons dans la Grande Guerre mais des Bretons et de la Grande Guerre. Nuance d'importance puisqu'au-delà de la simple chronique des années 1914-1918, ils posent la question du rapport des Bretons à la Mémoire de la guerre et à ses effets différés. Là se retrouve sans doute le mieux la spécificité régionale par la forte instrumentalisation mémorielle et politique de la guerre, spécialement sur la question, très sensible encore aujourd'hui, des pertes de guerre. Avec 140000 à 150000 décédés, soit près de $22 \%$ des mobilisés, la Bretagne demeure 
l'une des régions les plus durement touchées. Ce constat, justifié par la grande part de ruraux affectés à l'infanterie et par une démographie dynamique fortement pourvoyeuse de mobilisés, a suscité de nombreuses récupérations dans une région où le rapport à la mort est important. L'inflation du chiffre des morts, scellé dans la pierre de Sainte-Anne-d'Auray à 240000 et parfois gonflé jusqu'à 300000 , légitime les revendications des uns et des autres et l'idée d'une dette sacrée contractée par la France. Ce rappel au sang versé justifie la volonté de reconnaissance régionale d'une Bretagne catholique affichée par l'Église, celle d'une intégration nationale exprimée par les républicains ou le séparatisme revendiqué par les mouvements nationalistes. Ce n'est pas le moindre atout de cet ouvrage, ni le moins courageux, que d'en finir avec ce fantasme doloriste, victimaire et identitaire d'une Bretagne sacrifiée, vision reconstruite et légendaire aux visées propagandistes encore très présente malgré les progrès de la recherche. Au final, cet ouvrage de grande qualité, aussi bien sur la forme que sur le fond, comble un vide et apporte un éclairage intelligent et très accessible intégrant les recherches historiques les plus récentes. Si l'on nous permet de célébrer tout de même un petit particularisme breton, saluons donc la parution de ce livre qui distinguera dans les rayonnages le sérieux et la qualité de l'historiographie bretonne sur le sujet.

Ronan RICHARD

GEMIE, Sharif, Bretagne, la nation invisible (1750-1950), Spézet, Coop Breizh, 2013, $328 \mathrm{p}$.

Il y a tellement peu de traductions de l'historiographie anglo-saxonne, chacun étant aujourd'hui supposé lire couramment l'anglais, qu'on ne peut que se réjouir de l'initiative mettant opportunément à la disposition du public de langue française cet ouvrage sur la Bretagne, écrit par un universitaire gallois. Tout au plus peut-on regretter que l'opération, sans doute un peu précipitée, n'ait pas fait l'objet d'un ultime examen qui aurait permis de la débarrasser de quelques distractions factuelles, de quelques lourdeurs stylistiques, ou d'un certain nombre de coquilles que le lecteur est obligé, selon la formule consacrée, de corriger lui-même.

Le titre intrigue. Pourquoi donc la Bretagne serait-elle une nation " invisible "? Parce qu'on la cache? Parce qu'elle se cache? Les deux, finit-on par comprendre. En gros, le nationalisme breton s'est trouvé doublement empêché, contrarié dès le XIXe siècle par la politique jacobine des gouvernements français et délégitimé au milieu du $\mathrm{Xx}^{\mathrm{e}}$ par le dévoiement fasciste de ses éléments les plus radicaux. Il en a été réduit, après 1945, à se recomposer comme une simple identité sociale et culturelle, rejoignant ainsi aujourd'hui le courant du "néonationalisme ", au sein de ce qu'il est convenu d'appeler les minorités nationales. Mais dans la variante soft : celle qui n'éprouve plus le besoin de créer des frontières ni donc d'accéder au pouvoir d'État, à la différence de la variante hard, type flamand, qui y prétend encore. "Les enfants de Mordrel, conclut l'auteur, ont, dans leur très grande majorité, rejeté son déterminisme de la terre et du sang pour créer quelque chose de plus subtil, de mieux adapté à l'échelle humaine : ils habitent une nation invisible " (p. 292).

Avant de s'achever sur ce puissant raccourci, le livre entraîne le lecteur dans un curieux parcours, parfois déconcertant, alternant vastes panoramas et observations de détail, à travers deux siècles dont les bornes temporelles annoncées dans le titre indiquent un cadre général plus qu'un programme exhaustif ou un itinéraire chronologique. Le premier chapitre, largement nourri des travaux de 